\title{
Posterior Cramér-Rao Bound for Inertial Sensors Enhanced Mobile Positioning Under The Random Walk Motion Model
}

\author{
Ziming He, Yi Ma and Rahim Tafazolli
}

\begin{abstract}
This letter presents a new posterior Cramér-Rao bound (PCRB) for inertial sensors enhanced mobile positioning, which performs hybrid data fusion of parameters including position estimates, pedestrian step size, pedestrian heading, and the knowledge of random walk motion model. Moreover, a nonmatrix closed form of the PCRB is derived without position estimates. Finally, our numerical results show that when the accuracy of step size and heading measurements is high enough, the knowledge of random walk model becomes redundant.
\end{abstract}

Index Terms-Mobile positioning, inertial sensors, posterior Cramér-Rao bound (PCRB), random walk motion model.

\section{INTRODUCTION}

Inertial sensors enhanced mobile positioning refers to an advanced positioning technique that has potential of improving the accuracy through hybrid data fusion of parameters including radio positioning based position estimates, pedestrian step size, pedestrian heading, and the knowledge of motion models. The key idea is to combine the merits of radio positioning (such as satellites or mobile networks based techniques) and the foot-mounted inertial sensors (such as accelerometer and magnetometer) based pedestrian dead reckoning technique [1]. Radio positioning can be divided into non-cooperative and cooperative approaches [2]-[6]. The non-cooperative approaches only perform position-related parameter measurements between a mobile and several anchors ${ }^{1}$, while the cooperative approaches also perform the measurements between mobiles. One of the frequently utilized position-related parameters is distance, which can be obtained by received signal strength (RSS) and time-of-arrival (TOA) based ranging techniques [5][7]. The pedestrian dead reckoning technique can track the mobile position through inertial sensors, as illustrated in Fig. 1. Specifically, the pedestrian step size can be measured using an accelerometer, and the pedestrian heading can be measured using a magnetometer [1], [8]. The measurement is reasonably accurate when an inertial sensors empowered mobile terminal is put at an appropriate place such as in a trouser pocket [9]. Main shortcoming of the pedestrian dead reckoning technique is its introducing accumulative positioning errors to next state, which can be suppressed with the help of radio positioning techniques [1], [8]. In addition, the knowledge of motion models can further improve the positioning accuracy. One of the frequently used motion model is the random walk model [10]. The inertial sensor measurements and the knowledge

This work has been performed in the framework of the ICT project ICT248894 WHERE 2, which is partly funded by the European Union FP7 framework.

The authors are with Centre for Communication Systems Research, University of Surrey, Guildford, U.K., GU2 7XH. e-mail: y.ma@surrey.ac.uk.

${ }^{1}$ Anchors denote wireless devices whose positions are known. of motion models are also known as the information from temporal cooperation ${ }^{2}$ [4], [5].

Signal processing techniques employed for the hybrid data fusion are often model-based non-linear filters for instance Kalman filters or particle filters [5]. The parameters of interest for data fusion are classified into two categories: i.e., dynamic model (incl. motion models and inertial sensor measurements) and measurement model (position estimates from the radio positioning) (see [8], [11]). The former describes parameters reflecting correlation between adjacent states, and the latter describes parameters uncorrelated between states. An interesting research problem is to find posterior Cramér-Rao bound (PCRB) (see [12]) of the advanced positioning technique. Outcomes of our literature survey show that the PCRB has been analyzed for radio positioning techniques, for instance in [2], [3], radio positioning combined with the knowledge of motion models [10], radio positioning combined with both inertial sensor measurements and the knowledge of motion model under a general framework [4]. Moreover, the PCRB of radio positioning with either inertial sensor measurements or the knowledge of motion model is iteratively derived in [4] for each state by introducing the notion of carry-over information. The recursive derivation is important since it provide insights into the information evolution of positioning [4], [5].

Major contribution of this letter is to present a recursively derived PCRB for the positioning technique combining radio positioning based position estimates, inertial sensor measurements, and the knowledge of random walk model, which has not been addressed in the literature. This work is non-trivial since previous work in [4] is not applicable to the above positioning technique, where non-linear filters have to handle more than one dynamic models. Moreover, a non-matrix closed form of the PCRB is derived assuming no radio positioning based position estimates. Finally, our numerical results show that when the accuracy of inertial sensor measurements is high enough, the knowledge of random walk model becomes redundant.

Notations: Throughout the letter, we define $\nabla \mathbf{a} \triangleq$ $\left[\frac{\partial}{\partial a_{1}}, \ldots, \frac{\partial}{\partial a_{M}}\right]^{T}, \triangle_{\mathbf{b}}^{\mathbf{a}} \triangleq \nabla_{\mathbf{b}} \nabla_{\mathbf{a}}^{T}, p(\mathbf{a})$ the probability density function (p.d.f.) of the random vector $\mathbf{a},[\mathbf{a}]_{i}$ the $i$ th element in vector $\mathbf{a}, \otimes$ the Kronecker product, $\operatorname{Tr}\{\cdot\}$ the matrix trace.

\section{SYSTEM DESCRIPTION}

Fig. 1 depicts a mobile terminal (or a pedestrian) moving in sequence to $K$ different states step by step. The parameter of describing the $k$ th state is a vector $\boldsymbol{\theta}_{k} \triangleq\left[\begin{array}{ll}x_{k} & y_{k}\end{array}\right]^{T}$

\footnotetext{
${ }^{2}$ Temporal cooperation denotes a mobile infers its position by sharing information in the temporal domain [4].
} 


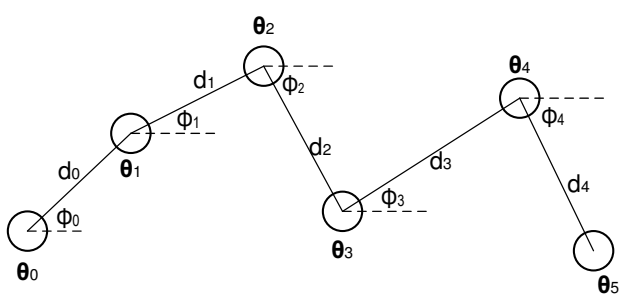

Fig. 1. Illustration of an example of the pedestrian dead reckoning: a mobile terminal (or a pedestrian) moves in sequence to positions at 6 different states $\boldsymbol{\theta}_{k}(k \in[0,5])$ step by step. The step size (i.e., distance $\left.d_{k}, k \in[0,4]\right)$ and the heading (i.e., angle $\phi_{k}, k \in[0,4]$ ) can be measured using inertial sensors. Provided that the position at initial state $\boldsymbol{\theta}_{0}$ is known, the positions at states $\boldsymbol{\theta}_{k}(k \in[1,5])$ can be determined, respectively.

( $k \in[0, K-1])$, where $x_{k}$ and $y_{k}$ denote the position. The total parameters can be collected to form a vector $\boldsymbol{\theta}=\left[\boldsymbol{\theta}_{0}^{T}, \boldsymbol{\theta}_{1}^{T}, \ldots, \boldsymbol{\theta}_{K-1}^{T}\right]^{T}$. Generally, the knowledge of random walk model can be modeled as a Gauss-Markov process [10]

$$
\boldsymbol{\theta}_{k+1}=\boldsymbol{\theta}_{k}+T_{k} \mathbf{w}_{k}
$$

where $T_{k}$ denotes the period between the $k$ th and the $(k+1)$ th state, i.e., step duration, $\mathbf{w}_{k}$ the constant speed between the $k$ th and the $(k+1)$ th state. $\mathbf{w}_{k}$ are modeled as uncorrelated zero-mean Gaussian noises with covariance matrix $\operatorname{diag}\left\{\omega_{x, k}^{2} \omega_{y, k}^{2}\right\}$, The step size measured using an accelerometer reads

$$
\hat{d}_{k}=d_{k}+v_{k}
$$

where

$$
d_{k}=\sqrt{\left(x_{k+1}-x_{k}\right)^{2}+\left(y_{k+1}-y_{k}\right)^{2}},
$$

$v_{k}$ are the measurement noises and modeled as uncorrelated zero-mean Gaussian variables with variances $\sigma_{k}^{2}$ [1]. $\hat{\mathbf{d}}$ is introduced to collect $\hat{d}_{k}$, given by $\hat{\mathbf{d}}=\left[\begin{array}{llll}\hat{d}_{0} & \hat{d}_{1} & \ldots & \hat{d}_{K-2}\end{array}\right]^{T}$. The heading measured using a magnetometer reads [1]

$$
\hat{\phi}_{k}=\phi_{k}+n_{k},
$$

where

$$
\phi_{k}=\tan ^{-1} \frac{y_{k+1}-y_{k}}{x_{k+1}-x_{k}},
$$

$n_{k}$ are uncorrelated zero-mean Gaussian variables with variances $\epsilon_{k}^{2} . \hat{\phi}$ is introduced to collect $\hat{\phi}_{k}$, given by $\hat{\phi}=$ $\left[\begin{array}{llll}\hat{\phi}_{0} & \hat{\phi}_{1} & \ldots & \hat{\phi}_{K-2}\end{array}\right]^{T}$, which is uncorrelated with $\hat{\mathbf{d}}$. Moreover, $\hat{\mathbf{d}}$ or $\hat{\phi}$ is uncorrelated with $\mathbf{w}_{k}$, respectively. At each state, the mobile terminal can obtain a radio positioning based estimate with p.d.f $p\left(\hat{\mathbf{r}}_{k} \mid \boldsymbol{\theta}_{k}\right)$, where $\hat{\mathbf{r}}_{k}$ denote the radio measurements of the position-related parameters. Define $\hat{\mathbf{r}} \triangleq$

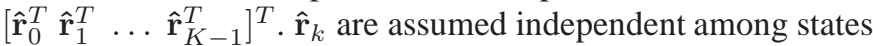
and un-correlated with $\hat{\mathbf{d}}, \hat{\phi}$ and $\mathbf{w}_{k}$. The positioning technique is to fuse $p\left(\hat{\mathbf{r}}_{k} \mid \boldsymbol{\theta}_{k}\right)$, the knowledge of motion model in (1), step size measurements in (2) and heading measurements in (4).

\section{ITERATIVE FORMULATION OF THE PCRB}

We start the derivation of the PCRB from the joint p.d.f as follow

$$
\begin{aligned}
p(\hat{\mathbf{r}}, \hat{\mathbf{d}}, \hat{\boldsymbol{\phi}}, \boldsymbol{\theta})= & p\left(\hat{\mathbf{r}}_{0} \mid \boldsymbol{\theta}_{0}\right) \prod_{k=0}^{K-2} p\left(\boldsymbol{\theta}_{k+1} \mid \boldsymbol{\theta}_{k}\right) p\left(\hat{d}_{k} \mid \boldsymbol{\theta}_{k+1}, \boldsymbol{\theta}_{k}\right)(6) \\
& p\left(\hat{\phi}_{k} \mid \boldsymbol{\theta}_{k+1}, \boldsymbol{\theta}_{k}\right) p\left(\hat{\mathbf{r}}_{k+1} \mid \boldsymbol{\theta}_{k+1}\right)
\end{aligned}
$$

The PCRB of estimating $\boldsymbol{\theta}$ can be expressed as

$$
\mathbb{E}_{\hat{\mathbf{r}}, \hat{\mathbf{d}}, \hat{\boldsymbol{\phi}}, \boldsymbol{\theta}}\left[(\hat{\boldsymbol{\theta}}-\boldsymbol{\theta})(\hat{\boldsymbol{\theta}}-\boldsymbol{\theta})^{T}\right] \geq \mathbf{J}(\boldsymbol{\theta})^{-1},
$$

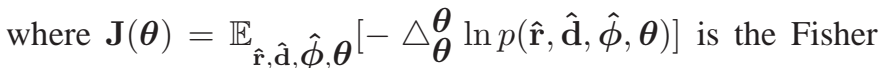
information matrix (FIM). Then, the problem is to formulate the information submatrix for estimating $\boldsymbol{\theta}_{k}$, which is denoted as $\mathbf{J}_{k}$. Once $\mathbf{J}_{k}$ is obtained, the square root of the PCRB of estimating position at the $k$ th state can be calculated as $\mathcal{P}_{k} \triangleq \sqrt{\operatorname{Tr}\left\{\left[\mathbf{J}_{k}^{-1}\right]\right\}}$.

It is found that the posterior information submatrix $\mathbf{J}_{k+1}$ for estimating vector $\boldsymbol{\theta}_{k+1}$ obeys the recursion (see the proof in Appendix A)

$$
\mathbf{J}_{k+1}=\boldsymbol{\Theta}_{k+1}+\left[\left(\mathbf{Q}_{k}^{-1}+\mathcal{H}_{k}\right)^{-1}+\mathbf{J}_{k}^{-1}\right]^{-1},
$$

where $\mathcal{H}_{k}$ is defined in (24). $\boldsymbol{\Theta}_{k+1}$ is defined in (17), and $\mathbf{Q}_{k} \triangleq T_{k}^{2} \operatorname{diag}\left\{\omega_{x, k}^{2} \omega_{y, k}^{2}\right\}$. Note that $\mathbf{J}_{0}=\boldsymbol{\Theta}_{0}$. (8) indicates that the posterior information of the $(k+1)$ th state is related to the posterior information of the $k$ th state, the radio positioning based estimates (i.e., $\boldsymbol{\Theta}_{k+1}$ ), the knowledge of step size and heading (i.e., $\mathcal{H}_{k}$ ) and the knowledge of random walk model (i.e., $\mathbf{Q}_{k}^{-1}$ ). For range-based radio positioning, $\boldsymbol{\Theta}_{k}$ $(k \in[0, K-1])$ can be written as [2], [10]

$$
\boldsymbol{\Theta}_{k}=\mathcal{G}_{k}^{T} \boldsymbol{\Lambda}_{k}^{-1} \mathcal{G}_{k},
$$

where $\mathcal{G}_{k}=\nabla_{\boldsymbol{\theta}_{k}}^{T} \otimes \mathbf{r}_{k}, \mathbf{r}_{k}$ a vector collecting the true distance between position $\boldsymbol{\theta}_{k}$ and the anchors, $\boldsymbol{\Lambda}_{k}=$ $\operatorname{diag}\left\{\lambda_{1, k} \lambda_{2, k} \ldots \lambda_{N, k}\right\}$ a diagonal matrix, $\lambda_{n, k}(n \in[1, N])$ the variance of range estimates of $\left[\mathbf{r}_{k}\right]_{n}$. For RSS-based ranging, $\lambda_{n, k}=\left(\xi\left[\mathbf{r}_{k}\right]_{n} \ln 10\right)^{2} /(10 \gamma)^{2}[10]$, where $\xi$ denotes the shadowing effect, $\gamma$ the path loss factor. For TOA-based ranging without channel knowledge, $\lambda_{n, k}=\left(c^{2}\right) /\left[8 \pi^{2} \beta^{2}(1-\right.$ $\left.\left.\chi_{n, k}\right) \mathrm{SNR}_{n, k}\right]$ [2], where $\chi_{n, k}(\in[0,1])$ denotes the pathoverlap coefficient, $\mathrm{SNR}_{n, k}$ the signal-to-noise-ratio of the line-of-sight path, $\beta$ the effective bandwidth, $c$ the light speed.

Without the knowledge of step size and heading, $\mathbf{H}_{k}^{11}, \mathbf{H}_{k}^{12}$, $\mathbf{H}_{k}^{22}$ in (18) vanished so that (8) reduces to

$$
\mathbf{J}_{k+1}=\boldsymbol{\Theta}_{k+1}+\left(\mathbf{Q}_{k}+\mathbf{J}_{k}^{-1}\right)^{-1},
$$

which has been shown in [10]. Without the knowledge of random walk model, $\mathbf{D}_{k}^{11}, \mathbf{D}_{k}^{12}, \mathbf{D}_{k}^{22}$ in (18) vanished so that (8) reduces to

$$
\mathbf{J}_{k+1}=\Theta_{k+1}+\left(\mathcal{H}_{k}^{-1}+\mathbf{J}_{k}^{-1}\right)^{-1} .
$$

(10) and (11) are special cases of the FIM derived in [4].

Without radio positioning based position estimates, i.e., $\Theta_{k}=\mathbf{0}$, the approach becomes the pedestrian dead reckoning w/o the knowledge of random walk model. In this case, the non-matrix closed form of the square root of the PCRB can be expressed as (see the proof in Appendix B)

$$
\begin{aligned}
& \mathcal{P}_{k+1}= \\
& \sqrt{\sum_{l=0}^{k} \frac{T_{l}^{2}\left[d_{l}^{2} \epsilon_{l}^{2} T_{l}^{2}+\sigma_{l}^{2} T_{l}^{2}+\sigma_{l}^{2} d_{l}^{2} \epsilon_{l}^{2}\left(\omega_{x, l}^{-2}+\omega_{y, l}^{-2}\right)\right]}{T_{l}^{4}+d_{l}^{2} \epsilon_{l}^{2} T_{l}^{2} \nu_{l}+\sigma_{l}^{2} T_{l}^{2} \mu_{l}+d_{l}^{2} \epsilon_{l}^{2} \sigma_{l}^{2} \omega_{x, l}^{-2} \omega_{y, l}^{-2}}+\mathcal{P}_{0}^{2}},
\end{aligned}
$$


where $\nu_{l}=\omega_{y, l}^{-2} \cos ^{2} \phi_{l}+\omega_{x, l}^{-2} \sin ^{2} \phi_{l}, \mu_{l}=\omega_{y, l}^{-2} \sin ^{2} \phi_{l}+$ $\omega_{x, l}^{-2} \cos ^{2} \phi_{l}$. The PCRB in (12) is a monotonically increasing function of $\sigma_{l}, \epsilon_{l}, \omega_{x, l}, \omega_{y, l}$ or $d_{l}$, respectively. Without the knowledge of random walk model, (12) reduces to

$$
\mathcal{P}_{k+1}=\sqrt{\sum_{l=0}^{k}\left(d_{l}^{2} \epsilon_{l}^{2}+\sigma_{l}^{2}\right)+\mathcal{P}_{0}^{2}} .
$$

It is observed from (12) and (13) that the positioning accuracy degrades as the index $k$ increases, which explains the phenomenon of accumulated position errors in the pedestrian dead reckoning [8]. With radio positioning based position estimates, the non-matrix closed form of the PCRB cannot be obtained. Thus, the PCRB is evaluated numerically in Section IV.

\section{Numerical Results AND Discussions}

We evaluate the accuracy of radio positioning using the mean square root of the PCRB defined by: $\frac{1}{\mathcal{I}} \sum_{i=1}^{\mathcal{I}} \mathcal{P}_{k}^{i}$, where $i$ denotes the index of Monte Carlo trial, and $\mathcal{I}$ the number of Monte Carlo trials ( $\mathcal{I}=1,000$ is used in this paper). We consider the cases w/o inertial sensor measurements and/or the knowledge of random walk model. There are 4 anchors with positions $\left[\begin{array}{ll}D & D\end{array}\right]^{T},\left[\begin{array}{ll}-D & D\end{array}\right]^{T},\left[\begin{array}{ll}D & -D\end{array}\right]^{T}$ and $\left[\begin{array}{ll}-D & -D\end{array}\right]^{T}$, respectively. The trajectory of the mobile are generated using (1) with $\boldsymbol{\theta}_{0}=\left[\begin{array}{ll}0 & 0\end{array}\right]^{T}, \omega_{x, k}=\omega_{y, k}=1 \mathrm{~m} / \mathrm{s} . \sigma_{k}=\eta d_{k}$, where $\eta$ is a ratio controlling the standard deviations, $\epsilon_{k}=\epsilon$, $T_{k}=0.5 \mathrm{~s}$. Specifically, Fig. 2 shows the RSS-based radio positioning, $D=10 \mathrm{~m}, \xi=3 \mathrm{~dB}, \gamma=4$. Fig. 3 shows the TOA-based radio positioning, $D=50 \mathrm{~m}, \chi_{n, k} \sim \mathcal{U}(0,1)$, $\mathrm{SNR}_{n, k} \sim \mathcal{U}(0,10) \mathrm{dB}, \beta=20 \mathrm{MHz}$.

The results plotted in Fig. 2 show: 1) with the full knowledge of random walk model and/or the inertial sensor measurements (i.e., with temporal cooperation), the PCRBs decrease as time increases (i.e., $k$ increases), which is the same with the results shown in [4]; 2) The positioning accuracy is direct proportional to the measurement accuracy of inertial sensors, for the cases w/o the knowledge of random walk model; 3) The full knowledge of random walk model can further improve the positioning accuracy. However, when the accuracy of sensor measurements is as high as for instance $\eta=10 \%, \epsilon=10^{\circ}$, the accuracy improvement due to the knowledge of random walk model can be ignored.

The results plotted in Fig. 3 show the positioning accuracy with different levels of random walk knowledge. The uncertainty of random walk model can be reflected from the matrix $\mathbf{Q}_{k}$ in (8). For examples, the full knowledge of random walk model means that we know exactly the elements $\left(\omega_{x, k}, \omega_{y, k}\right)$. When we have no knowledge of the random walk model, the elements $\left(\omega_{x, k}, \omega_{y, k}\right)$ in $\mathbf{Q}_{k}$ should be replaced by $+\infty$, and (8) reduces to (11). In order to show the effect of different levels of knowledge, we define a parameter $\varpi$ to replace the elements $\left(\omega_{x, k}, \omega_{y, k}\right)$ in $\mathbf{Q}_{k}$, and for sure we should have $\varpi \geq \omega_{x, k}, \varpi \geq \omega_{y, k}$. Fig. 3 shows that the knowledge decreases when $\varpi$ increases. It is observed that when $\varpi$ increases, the PCRBs with different levels of knowledge (solid lines) approach the PCRBs without the knowledge (dotted lines), for the cases without inertial sensor measurements and with different measurement accuracies of inertial sensors.

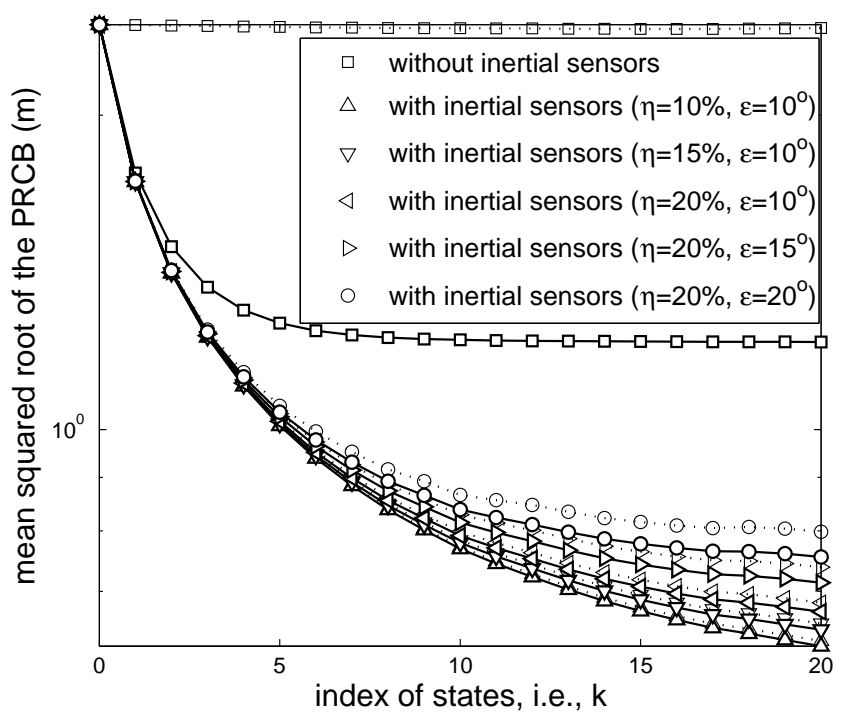

Fig. 2. Using RSS-based radio positioning, the mean square root of the PCRB as a function of the index of states. The dotted lines denote the PCRBs without the knowledge of random walk model, the solid lines denote the PCRBs with full knowledge of random walk model.

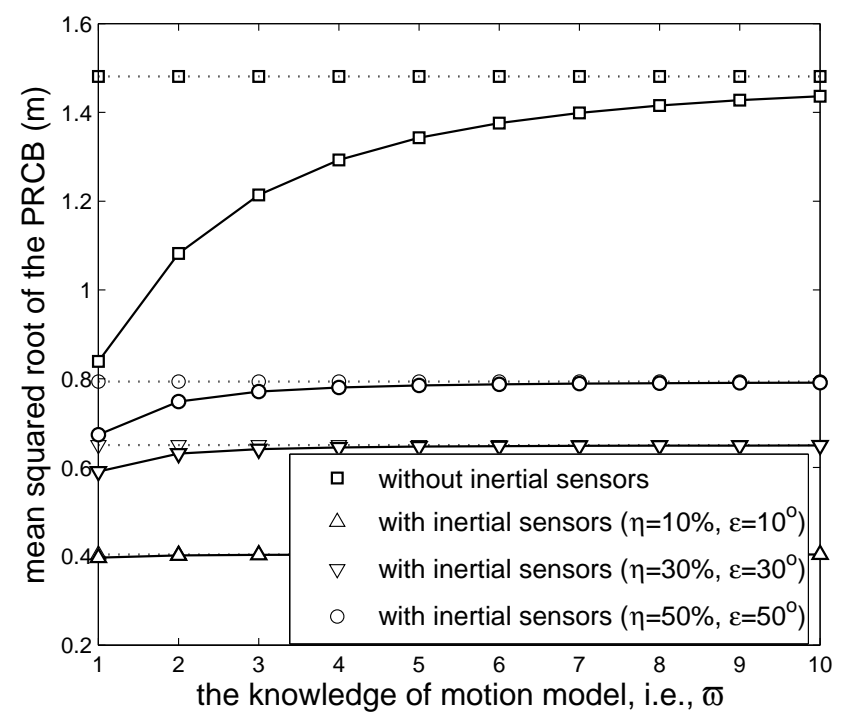

Fig. 3. Using TOA-based radio positioning, the mean square root of the PCRB at the 20th state as a function of different levels of the random walk knowledge. The dotted lines denote the PCRBs without the knowledge of random walk model, the solid lines denote the PCRBs with different levels of random walk knowledge.

\section{CONCLUSIONS}

This letter has presented a new PCRB for the advanced positioning technique combining radio positioning, pedestrian dead reckoning and the knowledge of random walk model. Moreover, a non-matrix closed form of the PCRB has been presented assuming no radio positioning. Finally, our numerical results have shown that when the accuracy of inertial sensor measurements is high enough, the knowledge of random walk 
model becomes redundant.

\section{APPENDIX A: ProOF OF (8)}

Define $p_{k} \triangleq p\left(\hat{\mathbf{r}}_{0: k}, \hat{\mathbf{d}}_{0: k-1}, \hat{\boldsymbol{\phi}}_{0: k-1}, \boldsymbol{\theta}_{0: k}\right)$, where $\hat{\mathbf{r}}_{0: k} \triangleq$ $\left[\begin{array}{llll}\hat{\mathbf{r}}_{0}^{T} & \hat{\mathbf{r}}_{1}^{T} & \ldots & \hat{\mathbf{r}}_{k}^{T}\end{array}\right]^{T}, \hat{\mathbf{d}}_{0: k-1} \triangleq\left[\begin{array}{llll}\hat{d}_{0} & \hat{d}_{1} & \ldots & \hat{d}_{k-1}\end{array}\right]^{T}, \hat{\boldsymbol{\phi}}_{0: k-1} \triangleq$ $\left[\begin{array}{llll}\hat{\phi}_{0} & \hat{\phi}_{1} & \ldots & \hat{\phi}_{k-1}\end{array}\right]^{T}, \boldsymbol{\theta}_{0: k+1} \triangleq\left[\begin{array}{llll}\boldsymbol{\theta}_{0}^{T} & \boldsymbol{\theta}_{1}^{T} & \ldots & \boldsymbol{\theta}_{k+1}^{T}\end{array}\right]^{T}$. By utilizing (6), $p_{k+1}$ can be written as

$p_{k} p\left(\hat{d}_{k} \mid \boldsymbol{\theta}_{k+1}, \boldsymbol{\theta}_{k}\right) p\left(\hat{\phi}_{k} \mid \boldsymbol{\theta}_{k+1}, \boldsymbol{\theta}_{k}\right) p\left(\boldsymbol{\theta}_{k+1} \mid \boldsymbol{\theta}_{k}\right) p\left(\hat{\mathbf{r}}_{k+1} \mid \boldsymbol{\theta}_{k+1}\right)$.

The FIM for $\boldsymbol{\theta}_{0: k}$ can be written as

$$
\begin{aligned}
& \mathbf{J}\left(\boldsymbol{\theta}_{0: k}\right) \\
& =\left[\begin{array}{ll}
\mathbb{E}\left(-\triangle \triangle_{0: k-1}^{\boldsymbol{\theta}_{0: k-1}} \ln p_{k}\right) & \mathbb{E}\left(-\triangle \triangle_{\boldsymbol{\theta}_{0: k-1}} \ln p_{k}\right) \\
\mathbb{E}\left(-\triangle \triangle_{0: k-1} \ln p_{k}\right) & \mathbb{E}\left(-\triangle \boldsymbol{\theta}_{k} \ln p_{k}\right)
\end{array}\right] \\
& \triangleq\left[\begin{array}{ll}
\mathbf{A}_{k} & \mathbf{B}_{k} \\
\mathbf{B}_{k}^{T} & \mathbf{C}_{k}
\end{array}\right] .
\end{aligned}
$$

Thus, $\mathbf{J}_{k}=\mathbf{C}_{k}-\mathbf{B}_{k}^{T} \mathbf{A}_{k}^{-1} \mathbf{B}_{k}$. Applying (14), the FIM for $\boldsymbol{\theta}_{0: k+1}$ can be written as

$$
\mathbf{J}\left(\boldsymbol{\theta}_{0: k+1}\right)=\left[\begin{array}{ccc}
\mathbf{A}_{k} & \mathbf{B}_{k} & \mathbf{0} \\
\mathbf{B}_{k}^{T} & \mathbf{C}_{k}+\mathbf{G}_{k}^{11} & \mathbf{G}_{k}^{12} \\
\mathbf{0} & \mathbf{G}_{k}^{21} & \boldsymbol{\Theta}_{k+1}+\mathbf{G}_{k}^{22}
\end{array}\right],
$$

where $\mathbf{0}$ denotes zero matrix with proper size,

$$
\begin{aligned}
& \boldsymbol{\Theta}_{k+1} \triangleq \mathbb{E}_{\hat{\mathbf{r}}, \boldsymbol{\theta}}\left[-\triangle_{\boldsymbol{\theta}_{k+1}}^{\boldsymbol{\theta}_{k+1}} \ln p\left(\hat{\mathbf{r}}_{k+1} \mid \boldsymbol{\theta}_{k+1}\right)\right], \\
& \left\{\begin{array}{ll}
\mathbf{G}_{k}^{11} \triangleq \mathbf{D}_{k}^{11}+\mathbf{H}_{k}^{11}, & \mathbf{G}_{k}^{12} \triangleq \mathbf{D}_{k}^{12}+\mathbf{H}_{k}^{12} \\
\mathbf{G}_{k}^{21} \triangleq\left(\mathbf{D}_{k}^{12}\right)^{T}+\left(\mathbf{H}_{k}^{12}\right)^{T}, & \mathbf{G}_{k}^{22} \triangleq \mathbf{D}_{k}^{22}+\mathbf{H}_{k}^{22}
\end{array},\right. \\
& \left\{\begin{array}{l}
\mathbf{D}_{k}^{11} \triangleq \mathbb{E}_{\boldsymbol{\theta}}\left[-\triangle_{\boldsymbol{\theta}_{k}}^{\boldsymbol{\theta}_{k}} \ln p\left(\boldsymbol{\theta}_{k+1} \mid \boldsymbol{\theta}_{k}\right)\right] \\
\mathbf{D}_{k}^{12} \triangleq \mathbb{E}_{\boldsymbol{\theta}}\left[-\triangle_{\boldsymbol{\theta}_{k+1}} \ln p\left(\boldsymbol{\theta}_{k+1} \mid \boldsymbol{\theta}_{k}\right)\right] \\
\mathbf{D}_{k}^{22} \triangleq \mathbb{E}_{\boldsymbol{\theta}}\left[-\Delta_{\boldsymbol{\theta}_{k+1}}^{\boldsymbol{\theta}_{k+1}} \ln p\left(\boldsymbol{\theta}_{k+1} \mid \boldsymbol{\theta}_{k}\right)\right]
\end{array},\right.
\end{aligned}
$$

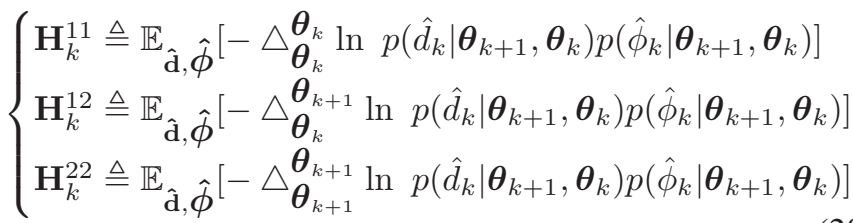

Thus, the posterior information submatrix for estimating $\boldsymbol{\theta}_{k+1}$ reads

$$
\begin{aligned}
\mathbf{J}_{k+1} & =\boldsymbol{\Theta}_{k+1}+\mathbf{G}_{k}^{22}-\left[\begin{array}{ll}
\mathbf{0} & \mathbf{G}_{k}^{21}
\end{array}\right]\left[\begin{array}{cc}
\mathbf{A}_{k} & \mathbf{B}_{k} \\
\mathbf{B}_{k}^{T} & \mathbf{C}_{k}+\mathbf{G}_{k}^{11}
\end{array}\right]^{-1}\left[\begin{array}{c}
\mathbf{0} \\
\mathbf{G}_{k}^{12}
\end{array}\right] \\
& =\boldsymbol{\Theta}_{k+1}+\mathbf{G}_{k}^{22}-\mathbf{G}_{k}^{21}\left(\mathbf{J}_{k}+\mathbf{G}_{k}^{11}\right)^{-1} \mathbf{G}_{k}^{12} .
\end{aligned}
$$

Based on the condition that the noise $\mathbf{w}_{k}$ in (1) is zero-mean Gaussian, it was shown in [12] that $\mathbf{D}_{k}^{11}=\mathbf{Q}_{k}^{-1}, \mathbf{D}_{k}^{22}=\mathbf{Q}_{k}^{-1}$, $\mathbf{D}_{k}^{12}=-\mathbf{Q}_{k}^{-1}$. Thus, (21) can be written as

$$
\begin{aligned}
\mathbf{J}_{k+1}= & \boldsymbol{\Theta}_{k+1}+\mathbf{Q}_{k}^{-1}+\mathbf{H}_{k}^{22}-\left(\mathbf{Q}_{k}^{-1}+\left(\mathbf{H}_{k}^{12}\right)^{T}\right) \\
& \left(\mathbf{J}_{k}+\mathbf{H}_{k}^{11}+\mathbf{Q}_{k}^{-1}\right)^{-1}\left(\mathbf{Q}_{k}^{-1}+\mathbf{H}_{k}^{12}\right) .
\end{aligned}
$$

Since the noise $v_{k}$ and $n_{k}$ are zero-mean Gaussian, $\mathbf{H}_{k}^{11}, \mathbf{H}_{k}^{12}$, $\mathbf{H}_{k}^{22}$ in (22) can be calculated as [13]

$$
\mathbf{H}_{k}^{11}=\mathbf{H}_{k}^{22}=-\mathbf{H}_{k}^{12}=\mathcal{H}_{k},
$$

where

$$
\begin{gathered}
\mathcal{H}_{k} \triangleq \frac{\mathbf{J}_{\hat{d}}\left(\phi_{k}\right)}{\sigma_{k}^{2}}+\frac{\mathbf{J}_{\phi}\left(\phi_{k}\right)}{\epsilon_{k}^{2} d_{k}^{2}}, \\
\mathbf{J}_{\hat{d}}\left(\phi_{k}\right)=\left[\begin{array}{cc}
\cos ^{2} \phi_{k} & \cos \phi_{k} \sin \phi_{k} \\
\cos \phi_{k} \sin \phi_{k} & \sin ^{2} \phi_{k}
\end{array}\right], \\
\mathbf{J}_{\hat{\phi}}\left(\phi_{k}\right)=\left[\begin{array}{cc}
\sin ^{2} \phi_{k} & -\cos \phi_{k} \sin \phi_{k} \\
-\cos \phi_{k} \sin \phi_{k} & \cos ^{2} \phi_{k}
\end{array}\right] .
\end{gathered}
$$

Using matrix analysis, (22) can be further expressed in (8).

APPENDIX B: PROOF OF (12)

Applying (8) and $\boldsymbol{\Theta}_{k}=\mathbf{0}$, we can obtain

$$
\mathcal{P}_{k+1}^{2}=\operatorname{Tr}\left\{\left(\mathcal{H}_{k}+\mathbf{Q}_{k}^{-1}\right)^{-1}\right\}+\mathcal{P}_{k}^{2},
$$

where

$$
\begin{aligned}
& \left(\mathcal{H}_{k}+\mathbf{Q}_{k}^{-1}\right)^{-1}= \\
& \rho_{k}\left[\begin{array}{cc}
f_{k} & \cos \phi_{k} \sin \phi_{k}\left(\sigma_{k}^{2}-d_{k}^{2} \epsilon_{k}^{2}\right) \\
\cos \phi_{k} \sin \phi_{k}\left(\sigma_{k}^{2}-d_{k}^{2} \epsilon_{k}^{2}\right) & g_{k}
\end{array}\right],
\end{aligned}
$$

$f_{k}=\left(d_{k}^{2} \epsilon_{k}^{2} T_{k}^{2} \sin ^{2} \phi_{k}+\sigma_{k}^{2} T_{k}^{2} \cos ^{2} \phi_{k}+\sigma_{k}^{2} d_{k}^{2} \epsilon_{k}^{2} \omega_{y, k}^{-2}\right) / T_{k}^{2}$, $g_{k}=\left(d_{k}^{2} \epsilon_{k}^{2} T_{k}^{2} \sin ^{2} \phi_{k}+\sigma_{k}^{2} T_{k}^{2} \cos ^{2} \phi_{k}+\sigma_{k}^{2} d_{k}^{2} \epsilon_{k}^{2} \omega_{y, k}^{-2}\right) / T_{k}^{2}$, $\rho_{k}=T_{k}^{4} /\left(T_{k}^{4}+d_{k}^{2} \epsilon_{k}^{2} T_{k}^{2} \nu_{k}+\sigma_{k}^{2} T_{k}^{2} \mu_{k}+d_{k}^{2} \epsilon_{k}^{2} \sigma_{k}^{2} \omega_{x, k}^{-2} \omega_{y, k}^{-2}\right), \nu_{k}=$ $\omega_{y, k}^{-2} \cos ^{2} \phi_{k}+\omega_{x, k}^{-2} \sin ^{2} \phi_{k}, \mu_{k}=\omega_{y, k}^{-2} \sin ^{2} \phi_{k}+\omega_{x, k}^{-2} \cos ^{2} \phi_{k}$. Thus, (12) can be obtained by applying (27) repeatedly.

\section{REFERENCES}

[1] R. Jirawimut, P. Ptasinski, V. Garaj, F. Cecelja, and W. Balachandran, "A method for dead reckoning parameter correction in pedestrian navigation system", IEEE Trans. Instrum. Meas., vol. 52, no. 1, pp. 209-215, Feb. 2003.

[2] Y. Shen and M. Z. Win, "Fundamental Limits of Wideband Localization - Part I: A General Framework", IEEE Trans. Inf. Theory, vol. 56, no. 10, pp. 4956-4980, Oct. 2010.

[3] Y. Shen, H. Wymeersch, and M. Z. Win, "Fundamental Limits of Wideband Localization - Part II: Cooperative Networks", IEEE Trans. Inf. Theory, vol. 56, no. 10, pp. 4981-5000, Oct. 2010.

[4] Y. Shen, S. Mazuelas, and M. Z. Win, "Cooperative Network Navigation: Fundamental Limit and its Geometrical Interpretation", IEEE J. Sel. Areas Commun., 2011. [Online]. Available: http://arxiv.org/abs/1112.3599.

[5] M. Z. Win, A. Conti, S. Mazuelas, Y. Shen, W. M. Gifford, D. Dardari, and M. Chiani, "Network Localization and Navigation via Cooperation", IEEE Commun. Mag., vol. 49, no. 5, pp. 56-62, May. 2011.

[6] A. Conti, M. Guerra, D. Dardari, N. Decarli, and M. Z. Win, "Network Experimentation for Cooperative Localization", IEEE J. Sel. Areas Commun., vol. 30, no. 2, pp. 467-475, Feb. 2012.

[7] D. Dardari, A. Conti, U. Ferner, A. Giorgetti, and M. Z. Win, "Ranging With Ultrawide Bandwidth Signals in Multipath Environments", Proc. IEEE, vol. 97, no. 2, pp. 404-426, Feb. 2009.

[8] A. R. J. Ruiz, F. S. Granja, J. C. P. Honorato, and J. I. G. Rosas, "Accurate pedestrian indoor navigation by tightly coupling foot-mounted IMU and RFID measurements", IEEE Trans. Instrum. Meas., vol. 61, no. 1, pp. 178-189, Jan. 2012.

[9] U. Steinhoff and B. Schiele, "Dead reckoning from the pocket - an experimental study", in Proc. IEEE Int. Conf. Perv. Comput. and Commun., Mar. 2010.

[10] F. Gustafsson and F. Gunnarsson, "Mobile positioning using wireless networks", IEEE Signal Process. Mag., vol. 22, no. 4, pp. 41-53, Jul. 2005.

[11] F. Gustafsson, F. Gunnarsson, N. Bergman, U. Forssell, J. Jansson, R. Karlsson, and P. J. Nordlund, "Particle filters for positioning, navigation and tracking", IEEE Trans. Signal Process., vol. 50, no. 2, pp. 425-437, Feb. 2002.

[12] P. Tichavský, C. H. Muravchik, and A. Nehorai, "Posterior cramérrao bounds for discrete-time nonlinear filtering", IEEE Trans. Signal Process., vol. 46, no. 5, pp. 1386-1396, May. 1998.

[13] H. L. Van Trees, Dectection, Estimation, and Modulation Theory-Part I, Hoboken, NJ: Wiley, 1968. 